\title{
O TEMA “EXPERIMENTOS VIRTUAIS" NOS ANAIS DOS EVENTOS BRASILEIROS DE ENSINO DE FÍSICA (2005 - 2014)
}

\section{THE THEME "VIRTUAL EXPERIMENTS" IN CONGRESS PROCEEDINGS OF EVENTS PHYSICS EDUCATION BRAZILIAN (2005 - 2014)}

\author{
Ivanderson Pereira da Silva \\ Universidade Federal de Alagoas / Campus Arapiraca - ivanderson@gmail.com
}

Alberto Tarrago Marcantonio da Silva

Universidade Federal de Alagoas / Instituto de Física - tarragomarcantonio@hotmail.com

\section{Resumo}

No presente estudo apresentamos um inventário dos trabalhos publicados nos anais do Simpósio Nacional de Ensino de Física (SNEF); do Encontro de Pesquisa em Ensino de Física (EPEF); e do Encontro Nacional de Pesquisa em Educação em Ciências (ENPEC), entre 2005 e 2014, e que enfocam a temática dos experimentos virtuais. Trata-se de uma pesquisa de levantamento, cujo principal objetivo foi apresentar o quadro das publicações que enfocam os experimentos virtuais em práticas de ensino de Física dos últimos dez anos. Foram considerados apenas os artigos publicados em Comunicações Orais. Num primeiro momento foram levantados todos os artigos relacionados com o uso de Tecnologias da Informação e Comunicação (TIC); posteriormente foram selecionados aqueles que discutiam TIC no Ensino de Física; por fim foram considerados os que estavam diretamente relacionados aos experimentos virtuais no ensino de Física. Como resultados, foi verificado um crescimento no volume de investigações publicadas nos anais do SNEF, EPEF e ENPEC, relacionadas ao tema de pesquisa no período de 2005 - 2012. A partir de 2013 foi possível perceber uma pequena queda no volume dessas produções nesses veículos de divulgação científica. No total, foram evidenciados 346 estudos que tratam dessa temática e que estão disponibilizados nessa obra.

Palavras-chave: Experimentos Virtuais, Laboratórios Virtuais, Ensino De Física

\begin{abstract}
In the present study we show an inventory of the works published in the annals of the National Symposium of Physics Teaching (SNEF); Of the Research Meeting in Physics Teaching (EPEF); And the National Encounter of Research in Education in Sciences (ENPEC), between 2005 and 2014, which focus on the theme of virtual experiments. It is a survey, whose main objective was to present the framework of publications that focus on
\end{abstract}


virtual experiments in physics teaching practices of the last ten years. Only articles published in Oral Communications were considered. At first, all articles related to the use of Information and Communication Technologies (ICT) were raised; Later, those who discussed ICT in Physics Teaching were selected; Finally were considered those that were directly related to the virtual experiments in the teaching of Physics. As a result, there was an increase in the volume of investigations published in the annals of SNEF, EPEF and ENPEC related to the research topic in the period 2005-2012. From 2013 onwards it was possible to perceive a small decrease in the volume of these productions in these vehicles scientific divulgation. In total, 346 studies were presented that address this theme and are available in this work.

Keywords: Virtual Experiments, Virtual Labs, Physical Education.

\section{Introdução}

Usar a experimentação como prática mediadora da construção do conhecimento é um dos grandes desafios da docência em Física na atualidade. Sua relação com o atual cenário tecnológico favorece a emergência de recursos de simulação que permitem aos sujeitos realizarem experimentos de Física a partir da tela do computador. Sob esse aspecto, as simulações virtuais, para o ensino de Física, vem ganhando cada vez mais espaço nas salas de aula. As tecnologias que possibilitam a utilização de simulações no ensino de Física, e de Ciências de um modo geral, vêm sendo analisadas pela comunidade científica há alguns anos. Nesse sentido, a questão central dessa pesquisa é a seguinte: "Como tem evoluído os estudos sobre os experimentos virtuais no âmbito das pesquisas em ensino de Física no período de 2005 a 2014?"

Essa investigação teve como objetivo principal verificar em qual estado se encontra a temática da experimentação virtual nos estudos publicados nos principais eventos da área do Ensino de Física. São eles: Encontro de Pesquisa em Ensino de Física (EPEF); Simpósio Nacional de Ensino de Física (EPEF) e Encontro de Pesquisa em Educação em Ciências (ENPEC). De modo específico, se objetiva compreender o que se tem discutido sobre a experimentação virtual e os laboratórios virtuais na área do ensino de Física, bem como mapear os estudos publicados nos principais eventos da área do Ensino de Física que enfocam essa temática.

Trata-se de uma pesquisa de levantamento na qual se evidenciam os artigos relacionados aos laboratórios virtuais de Física nos anais do SNEF, EPEF e ENPEC que ocorreram entre os anos de 2005 e 2014. O levantamento dos artigos relacionados com o tema foi feito em três etapas: 1) levantamento de todos os artigos relacionados ao uso de Tecnologias da Informação e da Comunicação (TIC); 2) Dentre os artigos que enfocavam o uso de TIC foram selecionados aqueles que tinham como foco o ensino de Física; 3) Selecionar dentre aqueles que exploravam o Ensino de Física por meio das TIC os que enfocavam a experimentação. A partir desse mapeamento foram classificados os estudos levantados de acordo com as categorias apontadas nessa revisão de literatura. 
Os resultados desse estudo foram organizados da seguinte forma: num primeiro momento, foi realizada uma discussão conceitual acerca dos experimentos, experimentos virtuais, laboratórios virtuais, bem como suas aplicações no cenário do ensino de Física presencial e a distância. As categorias de laboratórios e experimentos virtuais são apresentadas na sessão subsequente. A pesquisa de levantamento e a discussão sobre o tema sugerido, os resultados encontrados, os processos de consulta dos artigos expostos na internet e o passo a passo desse levantamento aparecem na última sessão.

\section{Experimentos e Experimentos Virtuais}

Para Souza (2002), um experimento é um procedimento planejado, partindo de uma hipótese, que visa provocar fenômenos em condições controladas, observar e analisar seus resultados. Segundo Giordan (1999) a experimentação caracteriza-se por despertar nos alunos, independentemente do nível de escolarização, a motivação para o estudo. Araújo e Abib (2003) classificam as atividades experimentais em: demonstração; verificação e investigação.

Na demonstração, o professor, como o único indivíduo ativo no processo, executa o experimento expondo aos alunos o fenômeno estudado. A experimentação como verificação favorece a confirmação de leis e teorias existentes na ciência. A experimentação como atividade de investigação favorece uma postura ativa para os alunos no processo de aprendizagem na qual poderão analisar, coletar dados e argumentar com seus colegas de sala sobre possíveis explicações para o fenômeno que estão explorando.

Pinho-Alves (2000), divide o conhecimento em duas vertentes: conhecimento vulgar ou senso comum e conhecimento científico. Em sua argumentação o autor discute os conceitos de "experiência" e "experimentação" a partir de seus antagonismos. Tais conceitos se contrapõem pela rigidez nos métodos utilizados. Nesse sentido,

A experiência está fortemente ligada ao cotidiano do ser humano, às suas interações mais livres e mais descomprometidas formalmente com o seu entorno socioambiental. Por outro lado vê-se que a experimentação está mais ligada ao homem investigador, àquele que busca organizar seus pensamentos na construção de elementos que lhe forneçam respostas sobre as coisas que o rodeiam e sobre si mesmo. A experiência é um fazer mais livre, um proceder fortemente guiado pela intuição no especular das coisas. A experimentação é um fazer elaborado, construído, negociado historicamente, que possibilita, através de processos internos próprios, estabelecer "verdades científicas" (PINHO-ALVES, 2000, p. 150).

Uma das grandes preocupações relacionadas ao ensino de Física no contexto da Educação Básica consiste em romper com os paradigmas que a rotulam como uma área de difícil compreensão. Os experimentos, que deveriam ser o pilar central do ensino das Ciências Experimentais, como é o caso da Física, passam a figurar como ferramentas alternativas na prática docente e nesse sentido concorrem para esse fenômeno as condições objetivas das escolas, do currículo e do trabalho docente. 
A experimentação no ensino de Física é de fundamental importância no processo ensino-aprendizagem e seu lugar na prática pedagógica pode se mover para diferentes campos do currículo. Para Alves e Stachak (2005, p.02), a experimentação no ensino de Física pode ser explorada "como ferramenta auxiliar ao processo ensino-aprendizagem ou como sendo o próprio processo da construção do conhecimento científico". Assim, o professor pode utilizar os experimentos como recurso didático para apoiar a prática do ensino dos conteúdos físicos ou mesmo utilizá-los como o próprio conteúdo de modo que os alunos ao aprender o experimento desenvolvam competências e habilidades investigativas.

Segundo Alves (2006), "quando o professor introduz os experimentos em sala de aula, ele se vê frente a um novo comportamento dos alunos: mais interessados e participativos. Neste momento, ele poderá optar por uma determinada didática que inclua o uso de atividades experimentais" (p. 15). Nesse sentido, atividades experimentais podem favorecer a motivação dos alunos para a aprendizagem de conceitos físicos, aprimorar a capacidade de observação e registro de informações e potencializar a aprendizagem de conceitos científicos (OLIVEIRA, 2010; GIORDAN, 1999).

A inserção do computador e de dispositivos móveis nas escolas como instrumentos didáticos vem ganhando espaço progressivamente e seus usos vem se tornando uma tendência mundial. Com o desenvolvimento das TIC e sua alta penetração social, a prática pedagógica passa a ser rediscutida à luz dos ganhos que tais recursos tecnológicos podem trazer para o fenômeno da aprendizagem. Dentre esses ganhos é possível apontar o advento de simulações que reproduzem laboratórios de Física e experimentos em ambientes virtuais (BISOL, 2010). Os laboratórios ou experimentos virtuais, historicamente recentes, surgiram da necessidade de adaptação dos laboratórios reais em um novo contexto capaz de atender ao aluno geograficamente distante (LIMA et al., 2005).

Atualmente contamos com uma gama enorme de softwares e aplicativos que simulam experimentos virtuais de Física. Dispositivos móveis, como os smartphones, disponibilizam aplicativos com alto grau de usabilidade, baseados na linguagem de programação em JAVA, que podem ser facilmente executados em quaisquer aparelhos e em qualquer hora do dia. Do mesmo modo, é possível encontrar computadores nas escolas de redes públicas e privadas que favorecem um amplo leque de possibilidades didáticas e que podem dar suporte à experimentação em Física. Os suportes digitais que tais recursos proporcionam, nos encaminham para o debate acerca da experimentação virtual. Alguns termos básicos precisam ser definidos para que possamos entender o que é um experimento virtual. Os conceitos de modelagem matemática e computacional irão contribuir para o entendimento do processo de construção, utilização de um experimento virtual.

Um modelo matemático é uma representação ou interpretação simplificada da realidade, ou uma interpretação de um fragmento de um sistema. O termo modelo foi introduzido na Matemática no último século e atualmente é amplamente utilizado no circuito acadêmico (MACINTYRE, 2002). Segundo Biembengut e Hein (2005), um conjunto de símbolos e relações matemáticas que traduz, de alguma forma, um fenômeno em questão ou um problema ligado a uma situação real, é denominado de modelo matemático. 
A modelagem computacional é a área que trata da simulação de soluções para problemas científicos, analisando os fenômenos, desenvolvendo modelos matemáticos para sua descrição e elaborando códigos computacionais para obtenção daquela solução. Já a simulação consiste em empregar técnicas matemáticas em computadores com 0 propósito de imitar um processo ou operação do mundo real. Desta forma, para ser realizada uma simulação é necessário construir um modelo computacional que corresponde à situação real que se deseja simular (FREITAS FILHO, 2008).

Coelho (2002) divide as simulações em dois grupos: interativas e não interativas. Nas simulações interativas, o usuário pode alterar vários parâmetros da simulação, explorando a situação física representada, verificando as implicações das alterações feitas no comportamento do fenômeno estudado (COELHO, 2002). Um exemplo de simulação interativa é a representada na figura 1.

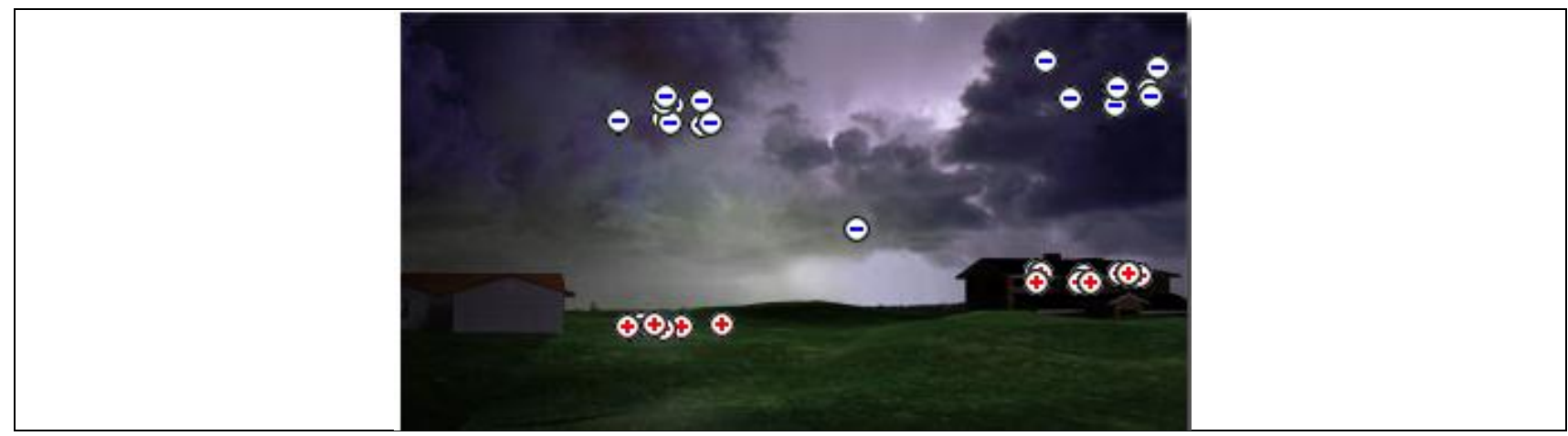

Figura 1 - Representação de uma simulação não interativa

Fonte: Disponível em: http://migre.me/uYtvv Acesso 20 mar 2016

O esquema representado na figura 1 pode ser comparado a um capacitor. No modelo proposto e simulado, as nuvens, carregadas negativamente, e o solo, carregado positivamente, criam uma diferença de potencial de tal magnitude que pode romper com a resistência dielétrica do ar e criar um meio condutor entre essas duas superfícies. Esse é o princípio de explicação da descarga elétrica e no caso supracitado, os raios. Nas simulações não interativas, o usuário não pode alterar os parâmetros da simulação. Segundo Heckler (2004, p.24), "os simuladores não interativos servem para mostrar e ilustrar a evolução temporal de algum evento ou fenômeno". Podemos verificar este tipo de simulação na figura 2 . 


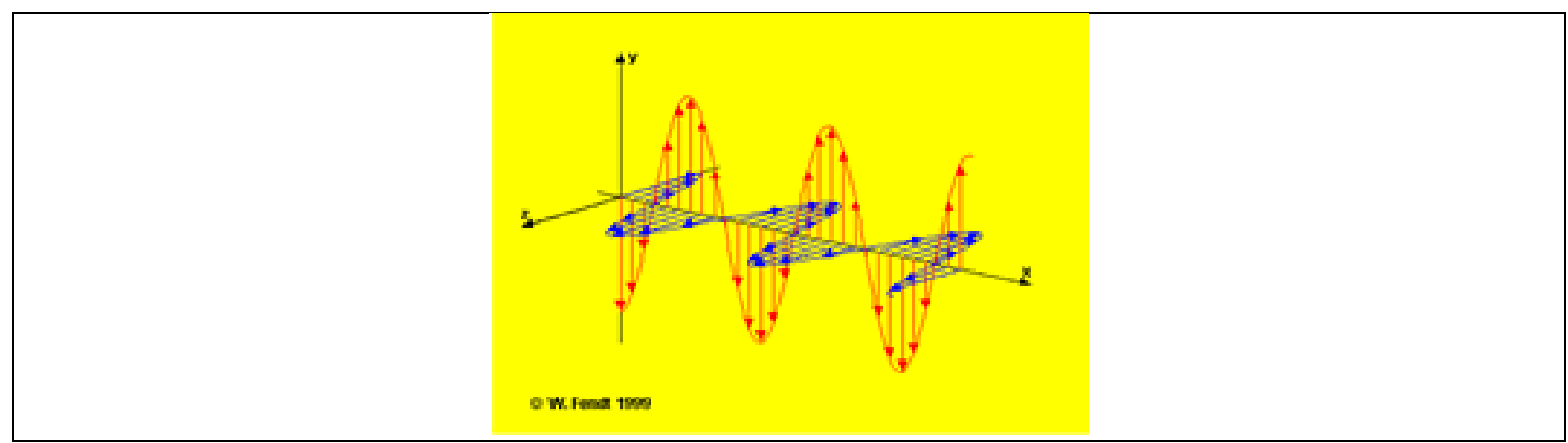

Figura 2 - Exemplo de uma simulação não interativa

Fonte: Disponível em: http://migre.me/uYtuH Acesso em 20 mar 2016

Na figura 2 é possível visualizar a representação de uma onda plana polarizada, se propagando na direção positiva do eixo $x$. Os vetores do campo elétrico (representados em vermelho) são paralelos ao eixo $y$, enquanto os vetores do campo magnético (representados em azul) são paralelos ao eixo $z$. Neste tipo de simulação podemos verificar a onda se propagando nas três dimensões, mas não podemos alterar nenhum parâmetro que provoque mudanças nas características da onda. Esse é um bom exemplo de simulação não interativa.

\section{Laboratórios Virtuais}

O conceito de laboratório virtual difere de autor para autor. Para Albu e Holbert (2004), um laboratório virtual pode ser definido como "ambiente de desenvolvimento interativo para criar e conduzir experimentos simulados". Fernandez et al. (2000), entendem que "o ambiente que permite o acompanhamento e controle de experimentos e ensaios remotamente é chamado de laboratório virtual". Já Álvares et al. (2003), afirmam que "os laboratórios virtuais podem ser uma bancada de testes de um sistema visando elaborar métodos para seu controle ou mesmo simulação de um sistema". Souza et al. (2001, p. 02), definem laboratórios virtuais como aqueles que "existem apenas nos arquivos de um sistema de computação, e, todos os seus equipamentos são desenvolvidos através de recursos de simulação, modelagem e visualização".

Os termos laboratórios virtuais e laboratórios de acesso remoto, apesar de considerados sinônimos, não são exatamente iguais. Os laboratórios virtuais usam programas para simular experiências. Eles são programas de computador que podem ser baixados da Internet e instalados num computador. Para utilizar o laboratório virtual, basta recorrer ao programa instalado no computador. Já os laboratórios de acesso remoto, são conectados, através da Internet, a equipamentos e instrumentos de um laboratório real. Neles, o sujeito manipula e controla, à distância, os equipamentos através de uma interface gráfica. Nos laboratórios virtuais, as imagens são filmes pré-produzidos e nos remotos, elas são geradas em tempo real de acordo com o experimento remotamente controlado. 
Os experimentos virtuais podem ser realizados em laboratórios virtuais de Física. Estes laboratórios consistem em um ambiente com diversas ferramentas digitais que possibilitam a interação entre aluno, professor e experimento. Para Guaita e Gonçalves (2014, p. 1465),

Os laboratórios virtuais simulam funções essências que estejam relacionadas em um determinado experimento, neste caso a condição física da atividade é substituída por um modelo computacional, cuja a base é um software de simulação. Há também a possibilidade de reproduzir um determinado experimento de maneira animada.

Muitos experimentos, como de eletricidade e de Física Moderna, tornam-se inviáveis pelo auto custo. É aí que a simulação virtual aparece como uma opção importante, possibilitando ao professor e ao aluno um contato com experimentos complexos e de difícil manuseio, como no caso dos experimentos de Física Quântica. Macêdo e Dickman (2009), mostram que estes problemas podem ser amenizados com o uso de simulações pois essas, possibilitam aos educandos observarem a evolução temporal de vários fenômenos em um tempo relativamente curto, permitindo ainda a manipulação dos dados e a repetição do experimento virtualmente a qualquer momento.

São várias as vantagens do uso dos experimentos virtuais no ensino de Física. Estes experimentos podem ser realizados em casa, salas de aulas e outros locais sem grande custo. Se o acesso aos computadores e à internet forem adequados é possível a realização da experiência a qualquer hora do dia, durante todos os dias do ano. Deste modo, os experimentos virtuais aparecem como uma alternativa para ausência de laboratórios de Ciências ou de experimentos que, por questões de segurança, espaço físicos, ou custo, tornam-se inviáveis de serem realizados fora do contexto da simulação computacional.

A experimentação virtual também carrega alguns riscos quanto se trata da veracidade das leis físicas. Um laboratório virtual produz experimentos que assentam, normalmente, em modelos. Se os modelos forem mal implementados, a simulação pode induzir o sujeito a erros conceituais. Outra desvantagem dos experimentos virtuais, encontra-se no contato que o aluno perde com os equipamentos. A coleta de dados, os resultados obtidos e as modelagens matemáticas são pré-estabelecidas pela máquina. Assim, o sujeito deixa de aprender com o erro e com as repetições dos experimentos. É claro que os experimentos virtuais são importantes, podem contribuir para a motivação dos alunos (MARTINHO; POMBO, 2009), mas não devem assumir um papel único em sala de aula. Deve aparecer como uma opção a mais para o professor e para o aluno. Lima et al. (2005, p.02), mostram que os experimentos virtuais acessados pela internet surgem como opção para democratização do ensino:

o desenvolvimento de laboratórios virtuais utilizando a internet como um meio capaz de interconectar espaços geográficos distintos surge como uma opção para as instituições de ensino, tornando mais democrática a utilização dos laboratórios existentes, promovendo o uso racional desses laboratórios e melhorando a qualidade da formação de seus alunos. 
Vários são as potencialidades didáticas dos experimentos virtuais para o ensino de Física. A cada ano que passa, novos softwares e aplicativos que simulam fenômenos físicos são criados e aperfeiçoados e estão ganhando mais espaço nas salas de aula, tanto em cursos presenciais quanto à distância. Não se tratam porém de substitutos dos experimentos reais, mas de complementos que podem desempenhar um papel que os experimentos reais jamais poderiam cumprir: o de favorecer a todos, todo tipo de experimentos didáticos.

\section{Categorias de laboratórios e experimentos virtuais}

Para Forte et al. (2008) os laboratórios virtuais podem ser caracterizados de acordo com o tipo de tecnologia aplicada: laboratórios multimídia, laboratórios em realidade virtual e laboratórios em realidade aumentada. Ou ainda por aspectos de colaboração: ambientes de colaboração local e ambientes de colaboração remota. Para esses autores,

Laboratórios Multimídia: é uma categoria de apoio ao aprendizado, de acesso fácil e amplo. Podem apresentar sons, textos, animações, vídeos e imagens, com o intuito de expor o conteúdo de maneira fácil e didática. São geralmente encontrados em Cds acompanhando algum material didático ou dispostos na internet. Um exemplo são os laboratórios virtuais do Genetic Science Learning Center da university of Utah.

Laboratórios em realidade virtual: neste tipo de laboratório, o usuário é inserido totalmente no mundo virtual através de ferramentas como óculos ou CAVE's (CaveAutomated Virtual Reality Environment). São laboratórios que se assemelham muito aos laboratórios reais e acabam sendo de alto investimento.

Laboratórios em realidade aumentada: diferente dos laboratórios de realidade virtual, os laboratórios de realidade aumentada (RA) não necessitam de uma imersão total e nem de recursos especiais de observação. A observação ocorre diretamente na tela do computador e o sujeito interage com os objetos virtuais com 0 auxilio de marcadores tangíveis. São estas aplicações que tornam este tipo de laboratório mais fácil de ser implementada. Tomemos como exemplo o LiDRA (Livro Didático com Realidade Aumentada), que pode ser um livro didático enriquecido com sons, imagens e objetos virtuais 3D.

Laboratórios virtuais remotos: "Um laboratório virtual remoto consiste de um laboratório real no qual os equipamentos são controlados remotamente, a partir de um computador conectado a web. A estrutura básica de um laboratório desse tipo é formada por um conjunto de instrumentos interfaceados a um computador. Esse computador tem a função de controlar, através de softwares específicos, o funcionamento dos equipamentos a ele interligados. Ele está conectado à web e, consequentemente, pode ser acessado a partir de outros computadores conectados à rede. O usuário remoto acessa e controla o computador do laboratório, aciona equipamentos, faz observações, testa condições e coleta dados. Câmaras de vídeo podem ser adicionadas ao ambiente do laboratório de modo que o usuário remoto consiga ter uma visão "online" do que se passa no laboratório real". (SOUZA et al., 2001, p. 03) 
Nos Laboratórios multimídia diferentes conceitos podem ser utilizados na exploração dos experimentos virtuais. Dentre esses conceitos é possível apontar os seguintes:

Objetos virtuais de aprendizagem (OVA): qualquer recurso digital que possa ser utilizado para suporte ao ensino (WILEY, 2000; SILVA, 2016). Esses recursos comumente são organizados em repositórios como o Banco Internacional de Objetos Educacionais (BIOE) <http://objetoseducacionais2.mec.gov.br/>, a Rede Interativa Virtual de Educação (RIVED) $\leq$ http://rived.proinfo.mec.gov.br> e 0 Portal do Professor do MEC $<$ http://portaldoprofessor.mec.gov.br/index.html>.

Software: são programas de computador que executam tarefas específicas e otimizam a manipulação de dados (JARDIM et. al., 2010; LUCENA et al., 2013). Existem uma ampla variedade de softwares que podem ser explorados patra suporte à prática pedagógica em Física. Nesse sentido, é possível citar o Celestia <http://www.shatters.net/celestia/>, 0 Stellarium $\leq$ http://www.stellarium.org/pt/>, e o Tabulae $\leq \mathrm{http}: / /$ tabulae.net $>$.

Imagens / Fotografias: as imagens ou as fotografias podem contribuir de forma significativa para a construção de experimentos baseados em recursos digitais. O professor pode explorar a câmera fotográfica (analógica e/ou digital), pode analisar ou produzir imagens com essas câmeras utilizando diferentes combinações de lentes e verificando os efeitos produzidos, pode ainda analisar as imagens a partir de tratamento com software adequado e realizar pesquisa com seus alunos como por exemplo no caso da análise do brilho de estrelas variáveis (CATELLI et al., 2010; COVERLONI et al., 2009; OURIQUE et al., 2010).

Simulação e animação: os termos simulação e animação frequentemente são utilizados indistintamente. No entanto, há de se destacar que o termo "simulação" deriva do latim "simulatus" que significa "imitar". Já o termo animação deriva do latim "animatio" que tem relação com a "qualidade do ser animado". A animação recupera a ideia do movimento, diferente da simulação que não necessariamente traz essa memória. A simulação evoca a ideia de mimese, representação, imitação, algo que imita ou que representa algo (SILVA, 2016). Uma técnica de animação que vem sendo utilizada com vistas à experimentação em Física é o stop motion, que pode ser "compreendido como movimentação pelo fenômeno da persistência retiniana, que provoca a ilusão no cérebro humano de que algo se move continuamente quando são reproduzidos mais de doze quadros por segundo" (FERREIRA, 2014, p. 745). No campo das simulações é possível encontrar na internet uma imensa variedade de repositórios como por exemplo o Phet Interactive Simulations <http://phet.colorado.edu/>.

Apllets: segundo Lucero et al. (2014, p. 109), são "programas que se pueden ejecutar directamente desde la página web en la que están insertos y se presentan como animaciones visuales con posibilidad de interacción por parte del usuario". Tratam-se de programas interativos, relativamente pequenos, configuráveis, que podem ser executados diretamente a partir da internet ou que podem ser facilmente recuperados de repositórios online. Um exemplo de repositório de Applets é o Java-Applets Zur Physic <http://www.walter-fendt.de/ph14e/>, que disponibiliza em 30 idiomas Applets de Física

Jogos digitais: Segundo Savi e Ulbricht (2008, p. 3), os jogos digitais são "como ambientes atraentes e interativos que do jogador ao oferecer desafios que exigem níveis crescentes 
de destreza e habilidades". Um exemplo é "O Guia do Físico das Galáxias", um jogo que envolve física e astronomia (CAMPOS et al., 2013). Outra experiência de desenvolvimento de jogos digitais para o ensino de Física é o projeto KS2 Bitesize. Desenvolvido pela British Broadcasting

Corporation <http://www.bbc.co.uk/schools/ks2bitesize/science/physical processes/>

Vídeos: Segundo Moran (1995 apud FERREIRA, 2014, p. 744), diferente das simulações, animações, applets, jogos digitais e softwares, a videogravação "parte do concreto, do visível, do imediato, próximo que troca todos os sentidos". Tais recursos têm sido utilizados como estratégias de sensibilização, demonstração, exploração, análise, iniciação científica, avaliação, bem como as variadas abordagens experimentais. Como exemplos de vídeos que podem dar suporte à experimentação em física é possível apontar os canais do Youtube

"Manual

do

Mundo"

<http://www.youtube.com/channel/UCKHhA5hN2UohhFDfNXB cvQ>

e "Mago da Física"

<http://www.youtube.com/channel/UCtw3Xw008t76Ns0M5kJJ09g>.

Áudio: $\mathrm{O}$ uso de dispositivos de captação, reprodução e análise de conteúdos em áudio têm se constituído numa linha de experimentação em Física. A exemplo disso é possível apontar as experiências desenvolvidas com o apoio do software Audacity. Trata-se de um software livre, de conteúdo aberto e que disponibiliza o download gratuito por meio do endereço: <<http://audacityteam.org/> Esse software auxilia a compreensão das características do som, como altura, intensidade e timbre, e de alguns fenômenos acústicos como batimento, reverberação e eco.

Modelagem computacional: A modelagem dos fenômenos contribui para definir a Física enquanto Ciência. Para Heidemann et al. (2012, p. 969), "a Física pode ser caracterizada como uma complexa rede de modelos interligados por um sistema de princípios teóricos", dentre os quais é possível destacar o modelo matemático. Quando a modelagem do fenômeno exige um tratamento matemático muito intenso ou complexo, é possível explorar os ambientes de modelagem computacional (AMC) com vistas à simulação desses modelos em ambientes virtuais de aprendizagem (AVA). Como exemplos desses ambientes é possível citar 0 software $<$ http://modellus.co/index.php $>; \quad 0 \quad$ SQrLab $<$ http://sqrlab.modelab.org>; e o Modelab2 <http://modelab2.modelab.org/>

Tecnologias móveis: outra tendência na experimentação contemporânea é o uso de dispositivos móveis para controlar experimentos à distância ou para explorar experimentos virtuais. Simão et al. (2013) apresentam o relato de uma experiência do desenvolvimento de uma interface de controle um experimento físico, observando os resultados via streaming de vídeo por meio de dispositivos móveis, o RExMobile. O aplicativo que controla os experimentos remotos, desenvolvidos por esses autores, se encontra disponível no link: $<$ http://rexlab.ufsc.br/experimentos/mobile $>$. Outro exemplo de uso de dispositivos móveis para suporte à experimentação em Física é a exploração de aplicativos para smartphone. Um dos recursos para smartphone disponíveis no Google Play é o LabVetor que, segundo Honorato et al. (2015, p.2), "é um aplicativo para dispositivos móveis que atua como uma ferramenta visando ao incremento dos recursos didáticos no ensino da Física e, em alguns casos, da Matemática". Tem por objetivo "ser uma ferramenta para auxiliar no ensino dos 
conceitos de vetores e grandezas vetoriais de modo a concretizar um pouco da sua abstração ao simular suas propriedades" (idem).

Hipermídia: Para Negroponte (1995, p. 66), hipermídia pode ser entendida como "um desenvolvimento do hipertexto, designando a narrativa com alto grau de interconexão, [...] [é como] uma coletânea de mensagens elásticas que podem ser esticadas ou encolhidas de acordo com as ações do leitor". É um recurso para o qual convergem diferentes formatos midiáticos tais como o impresso, o rádio, o vídeo e o hipertexto. Além de congregarem as potencialidades de cada um dos formatos midiáticos presentes em seu interior, a hipermídia, ao estabelecer a relação entre esses diferentes formatos midiáticos, proporciona experiências pedagógicas únicas.

Ambiente Virtual de Aprendizagem (AVA): Segundo Anjos (2012, p. 54), o AVA pode ser definido como "uma ou mais soluções de comunicação, gestão e aprendizado eletrônico, que possibilitam o desenvolvimento, integração e a utilização de conteúdos, mídias e estratégias de ensino-aprendizagem, a partir de experiências que possuem ou não referência com o mundo real e são virtualmente criadas ou adaptadas para propósitos educacionais". Trata-se de um espaço virtual onde são disponibilizados materiais que podem ser acessados por alunos de um curso ou disciplina. Este tipo de tecnologia permite a interação à distância entre professores, tutores, alunos, gestores, conteudistas, programadores e pessoal de suporte técnico. Como por exemplo de AVA é possível citar a plataforma Moddle, o Teleduc e o e-Proinfo.

Diante dessas categorias e da necessidade de traçar um panorama do estado em que as pesquisas sobre a experimentação virtual em Física se encontram na atualidade, foi realizado um levantamento nos anais do EPEF, SNEF e ENPEC realizados no período de 2005 a 2014. Os resultados desse levantamento estão disponíveis na sessão seguinte.

\section{A pesquisa de levantamento}

Para verificar em qual estado se encontrava o tema "experimentos virtuais" foi feito um levantamento dividido em três etapas: 1) levantamento das comunicações orais relacionadas ao uso de TIC publicadas nos anais dos eventos; 2) dentre os artigos que enfocavam o uso de TIC foram selecionados aqueles que tinham como foco o ensino de Física; 3) e dentre aqueles que exploravam o Ensino de Física por meio das TIC, foram selecionados os que enfocavam a experimentação virtual. O resultado desse levantamento foi organizado na tabela 1 que relaciona o número de artigos levantados em cada um dos anais e o ano de sua publicação. 


\begin{tabular}{|c|c|c|c|c|c|c|c|c|c|c|c|c|c|c|c|c|c|}
\hline & & \multicolumn{2}{|c|}{2005} & \multirow{2}{*}{$\begin{array}{l}2006 \\
\text { EPEF } \\
\end{array}$} & \multicolumn{2}{|c|}{2007} & \multirow{2}{*}{$\begin{array}{l}2008 \\
\text { EPEF }\end{array}$} & \multicolumn{2}{|c|}{2009} & \multirow{2}{*}{$\begin{array}{l}2010 \\
\text { EPEF }\end{array}$} & \multicolumn{2}{|c|}{2011} & \multirow{2}{*}{$\begin{array}{r}2012 \\
\text { EPEF }\end{array}$} & \multicolumn{2}{|c|}{2013} & \multirow{2}{*}{$\begin{array}{l}2014 \\
\text { EPEF }\end{array}$} & \multirow[t]{2}{*}{ Total } \\
\hline & & SNEF & ENPEC & & SNEF & ENPEC & & SNEF & ENPEC & & SNEF & ENPEC & & SNEF & ENPEC & & \\
\hline \multirow{12}{*}{$\begin{array}{l}\text { Laboratórios } \\
\text { Virtuais } \\
\text { Multimídia }\end{array}$} & OVA & 2 & 1 & 0 & 2 & 0 & 0 & 2 & 2 & 1 & 2 & 0 & 1 & 4 & 0 & 0 & 17 \\
\hline & Software & 4 & 5 & 0 & 4 & 1 & 0 & 0 & 2 & 3 & 5 & 0 & 3 & 2 & 1 & 0 & 30 \\
\hline & Imagem & 1 & 0 & 0 & 0 & 0 & 0 & 0 & 1 & 0 & 0 & 1 & 0 & 1 & 1 & 0 & 5 \\
\hline & $\begin{array}{l}\text { Simulação e } \\
\text { Animacão }\end{array}$ & 6 & 5 & 0 & 1 & 1 & 4 & 3 & 0 & 0 & 3 & 5 & 1 & 4 & 3 & 0 & 36 \\
\hline & Apllets & 0 & 0 & 0 & 0 & 0 & 0 & 1 & 1 & 0 & 0 & 0 & 0 & 0 & 0 & 0 & 2 \\
\hline & Jogos & 0 & 0 & 0 & 1 & 0 & 0 & 0 & 0 & 0 & 0 & 2 & 0 & 0 & 1 & 0 & 4 \\
\hline & Vídeos & 4 & 0 & 0 & 1 & 1 & 1 & 1 & 1 & 2 & 5 & 9 & 0 & 8 & 6 & 3 & 42 \\
\hline & Áudio & 0 & 0 & 0 & 0 & 0 & 0 & 1 & 0 & 0 & 0 & 0 & 0 & 0 & 0 & 0 & 1 \\
\hline & $\begin{array}{l}\text { Modelagem } \\
\text { computacional }\end{array}$ & 2 & 2 & 1 & 1 & 2 & 1 & 2 & 0 & 1 & 2 & 1 & 0 & 1 & 1 & 1 & 18 \\
\hline & $\begin{array}{l}\text { Tecnologias } \\
\text { Móveis }\end{array}$ & 0 & 0 & 0 & 0 & 0 & 0 & 0 & 0 & 0 & 0 & 1 & 0 & 1 & 0 & 0 & 2 \\
\hline & Hipermídia & 0 & 1 & 1 & 1 & 2 & 0 & 2 & 0 & 0 & 3 & 2 & 0 & 0 & 1 & 0 & 13 \\
\hline & AVA & 3 & 2 & 1 & 5 & 1 & 1 & 3 & 1 & 1 & 3 & 0 & 2 & 3 & 2 & 0 & 28 \\
\hline
\end{tabular}

Laboratório

em

Realidade

Virtual

Laboratório

de

Realidade

Aumentada

Laboratórios

Remotos

Outros

Total

\begin{tabular}{|c|c|c|c|c|c|c|c|c|c|c|c|c|c|c|c|}
\hline 7 & 3 & 1 & 9 & 3 & 2 & 14 & 5 & 4 & 20 & 14 & 10 & 15 & 15 & 9 & 131 \\
\hline 29 & 20 & 4 & 26 & 11 & 10 & 31 & 13 & 12 & 44 & 40 & 18 & 43 & 32 & 13 & 346 \\
\hline
\end{tabular}

Tabela 1 - Conceitos para a discussão sobre a experimentação virtual em Física 
A visualização da Tabela 1 nos permite verificar que é muito baixa a produção que enfoca a experimentação baseada em realidade virtual e na realidade aumentada bem como a experimentação remota. Com relação à experimentação baseada em laboratórios multimídia, os principais conceitos explorados foram os de software, simulação e vídeo. Esses conceitos totalizam quase um terço de toda a produção na área durante esse período.

Observa-se que a realidade aumentada é um conceito que quase não é discutido nos estudos que enfocam o uso de TIC no ensino de Física tendo sido encontrado em apenas um único estudo publicado no ENPEC de 2011. Verifica-se também que dos 346 artigos encontrados, 131 foram classificados como "outros". Foram enquadrados nessa categoria todos os estudos que discutiam práticas experimentais com o uso de TIC e que não estavam previstas na literatura ou predominantemente aquelas que faziam reflexões teóricas sobre o tema em tela. Trata-se de um número expressivo de discussões enfocando essa categoria.

Nota-se que os anos de 2011 e 2013 são os anos com o maior número de publicações no SNEF e no ENPEC. No SNEF 2011, 44 artigos foram publicados, sendo a maioria deles relacionados ao uso de software (05 publicações), vídeos (05 publicações) e outros (20 publicações). Já no ENPEC do mesmo ano, 40 artigos foram publicados sendo a maioria relacionados ao uso de Vídeos (09 publicações) e outros (14 publicações). Em 2013, o SNEF teve 43 artigos publicados, sendo 08 publicações referentes ao uso de vídeos, 04 referentes ao uso de OVA e 04 com o uso de animação e simulação. A categoria outros aparece também com 15 publicações, mesmo número do ENPEC 2013, que também teve a categoria vídeos como o maior número de publicações (06 publicações).

O EPEF é o encontro com o menor número de publicações entre os três. Em 2006 verificou-se apenas 04 publicações referentes ao tema, sendo relacionadas a modelagem computacional, hipermídia, AVA e outros; cada uma com uma publicação apenas. Já em 2012, verifica-se uma quantidade maior de trabalhos. Sendo software (03 publicações) e outros (10 publicações) as categorias com maior número de publicações. Observa-se pelo Gráfico 1 que o volume de estudos acerca desse tema tem crescido em todos os eventos estudados. No entanto, nos últimos eventos observa-se uma pequena queda a partir de 2013, dado que sugere uma possível perda de interesse pelo tema, implicando um novo estudo para verificar tal fenômeno.

Ao transpor os dados da tabela 1 para o Gráfico 1, é possível verificar com mais clareza a evolução do volume de publicação em função do tempo.

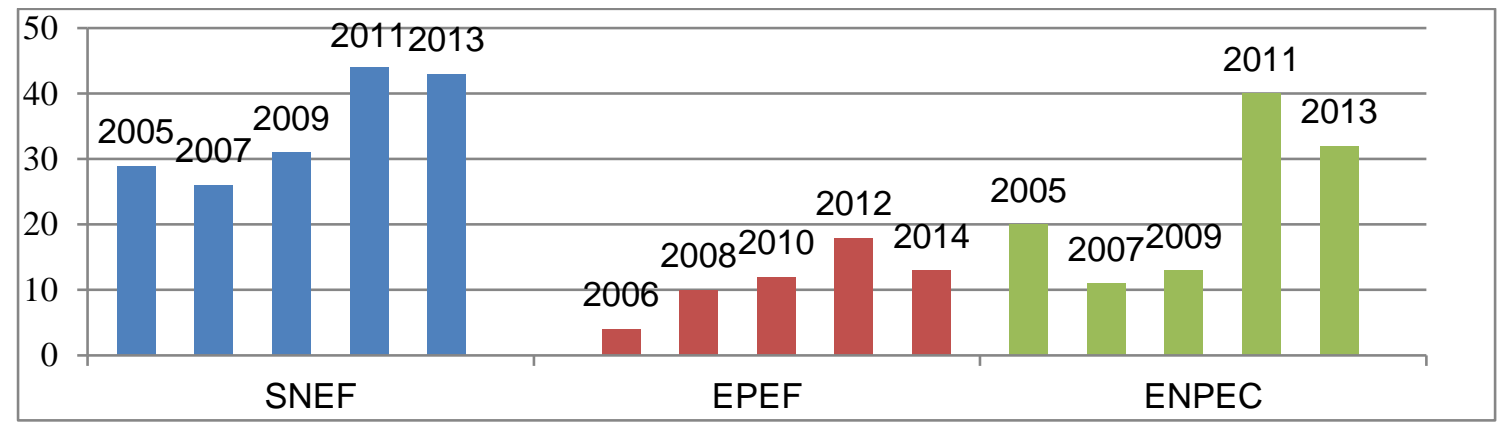

Gráfico 1 - Artigos publicados nos anais do SNEF, EPEF e ENPEC que discutiram acerca dos experimentos virtuais no período de $2005-2014$ 
A visualização do gráfico 1 nos permite perceber um crescimento no número de publicações que enfocam a experimentação virtual em Física entre 2005 e 2012. Do mesmo modo, percebemos que a partir de 2013 o volume desses trabalhos teve uma queda sutil do número absoluto de trabalhos com esse foco em relação à sua edição anterior. Como esse levantamento se concentrou no intervalo de 2005 - 2014 não é possível afirmar que essa queda represente uma tendência nas pesquisas dessa área.

\section{Considerações Finais}

Entre os anos de 2005 e 2014 as publicações referentes aos experimentos virtuais cresceram significativamente acompanhando a evolução tecnológica de uma maneira geral. Com uma ampla gama de possibilidades que o tema sugere, outros estudos relacionados ao uso de TIC nas aulas de Física surgem como possibilidade de investigação. Outros temas relacionados ao uso das TIC nas aulas aparecem como opção de estudos relacionados ao tema, dentre os quais o uso de tecnologias móveis como apoio à experimentação em Física. Muitos aplicativos que envolvem os experimentos virtuais estão sendo desenvolvidos e disponibilizados na internet e podem ser explorados nas escolas e universidades.

Observamos que esse é um tema que apresentou crescimento no volume de trabalhos apresentados nos principais eventos da área entre os anos de $2005-2012$. Diferentes hipóteses podem ser levantadas para justificar uma aparente queda no volume desses trabalhos nesses meios de divulgação científica: o volume total de trabalhos publicados nos anais desses eventos pode ter reduzido e proporcionalmente o número de trabalhos com foco na experimentação virtual pode ter acompanhado essa redução; a redução pode ser sido um fenômeno pontual desse período e após o ano de 2014 o número de trabalhos voltados para a experimentação virtual em física pode ter voltado a crescer; fatores relacionados à recente crise econômica cíclica mundial, que atinge seu ápice em 2012, podem ter contribuído para que menos sujeitos participassem dos eventos, dentre outros. Esses são apontamentos para novos estudos.

Outras sugestões para novas investigações podem ser apontadas a partir das carências identificadas nos estudos que pesquisam a experimentação baseada em realidade virtual e em realidade aumentada. Tais estudos são fundamentais para a consolidação desse, como um campo de pesquisa e naturalmente para ampliar as possibilidades didáticas para a prática pedagógica dos professores. A lista com as referências dos estudos levantados está disponível no link http://migre.me/uYsPt e pode contribuir para que os pesquisadores interessados nesse tipo de tema possa encontrar rapidamente um material de boa qualidade para fundamentar e iluminar os seus percursos teórico-metodológicos. 


\section{Referências}

ALBU M. M.; HOLBERT, K. E. Embedding Remote Experimentation in Power Engineering Education. IEEE Transactions On Power Systems, v. 19, n 1, 2004. Disponível em: http://migre.me/uYu26 Acesso em 13 set 2016.

ÁLVARES, A. J.; et al. Metodologia para Implantação de Laboratórios Remotos via Internet na Área de automação da Manufatura. Em: Il Congresso Brasileiro de Engenharia de Fabricação, 2003, Anais... Uberlândia/MG: UFU, 18-21 de Maio de 2003. Disponível em: http://migre.me/uYu9g Acesso em 20 mar 2016

ALVES, V. C.; STACHAK, M. A importância de aulas experimentais no processo ensino aprendizagem em Física: "eletricidade". Em XVI Simpósio Nacional de Ensino de Física, 2005, Anais... Rio de Janeiro/RJ: CEFET-RJ, 24 a 28 de Janeiro de 2005. Disponível em: http://migre.me/uYuom Acesso em 25 jan 2016.

ALVES, V. F. A inserção de atividades experimentais no ensino de Física em nível médio: em busca de melhores resultados de aprendizagens. Universidade de Brasília. Decanato de pesquisa e Pós-graduação. Instituto de Física. Instituto de Química. Programa de Pós-graduação em Ensino de Ciências. Mestrado em Ensino de Ciências. 2006. Disponível em: http://migre.me/uYupD Acesso em 25 jan 2016.

ANJOS, A. M. Tecnologias da informação e da comunicação, aprendizado eletrônico e ambientes virtuais de aprendizagem. In: MACIEL, C. (Org.) Ambientes Virtuais de Aprendizagem. Cuiabá-MT: EDUFMT, 2012.

ARAÚJO, M. S.; ABIB, M. L. Atividades Experimentais no Ensino de Física: diferentes enfoques, diferentes finalidades. Revista Brasileira de Ensino de Física, v. 25, no 2, p. 176-194, 2003. Disponível em: http://migre.me/uYuqJ Acesso em 25 jan 2016.

BIEMBENGUT, M. S.; HEIN, N. Modelagem matemática no ensino. São Paulo: Contexto, 2005. $128 \mathrm{p}$.

BISOL, C. A. Aprendizagem em ambientes virtuais: compartilhando ideias e construindo cenários. $2^{\circ}$ Ed. Caxias do Sul, RS: Educs, 2010. Disponível em: http://migre.me/uYurh Acesso em 20 mar 2016.

CAMPOS, F. C. et al. O guia do físico das galáxias: um jogo virtual para o ensino de física por meio de conceitos de astronomia. In: SIMPÓSIO NACIONAL DE EDUCAÇÃO EM ASTRONOMIA, 3, 2013, Curitiba. Anais... Curitiba: Universidade Tecnológica Federal do Paraná, 2013. $\quad$ p. $\quad 1$ - $2 . \quad$ Disponível em: http://snea2012.vitis.uspnet.usp.br/snea3/sites/default/files/SNEA2014 TCP67.pdf Acesso em: 9 ago. 2015.

CATELLI, F. et al. Um estudo de cinemática com câmara digital. Revista Brasileira de Ensino de Física, v. 32, n. 1, 2010. Disponível em: http://www.scielo.br/pdf/rbef/v32n1/a15 v32n1.pdf Acesso em: 21 jun. 2016. 
COELHO, R. O. O uso da informática no ensino de física de nível médio. Dissertação de Mestrado em Educação, Faculdade de Educação da Universidade Federal de Pelotas, UFPel, Pelotas. 2002.

COVERLONI, E. P. et al. Utilização de máquina fotográfica digital (multi-burst) para aulas experimentais de cinemática - queda livre. Revista Brasileira de Ensino de Física, v. 31, n. 3, 2009. Disponível em: http://www.sbfisica.org.br/rbef/pdf/313504.pdf Acesso em: 9 ago. 2015.

FERNANDEZ, R. O.; et al. Laboratório Virtual Aplicado à Educação a Distância. Em: XI Simpósio Brasileiro de Informática na Educação, 2000, Anais... Maceió/AL: UFAL, 2000. Disponível em: http://migre.me/uYuBL Acesso em 20 mar 2016.

FERREIRA, J. C. A produção de vídeos no ensino de ciências: o professor-autor e as tecnologias digitais. In: CONGRESSO INTERNACIONAL DAS TIC NA EDUCAÇÃO, 3., 2014, Lisboa. Anais... Lisboa: Instituto da Educação da Universidade de Lisboa, 2014. p. 742-747. Disponível em: http://ticeduca2014.ie.ul.pt/downloads/AtasDigitais/Atas Digitais ticEDUCA2014.pdf Acesso em: 9 ago. 2015.

FORTE, C.; et al. Implementação de Laboratórios Virtuais em Realidade Aumentada para Educação à Distância. Em: 5o Workshop de Realidade Virtual e Aumentada, 2008, Anais... Bauru/SP: UNESP, 19 a 21 de Novembro de 2008. Disponível em: http://migre.me/uYuDh Acesso em 25 jan 2016.

FREITAS FILHO, P. J. Introdução à Modelagem e Simulação de Sistemas: com Aplicações em Arena. 2. ed. Florianópolis: Visual Books Ltda, 2008.

GIORDAN, M. O papel da experimentação no ensino de ciências. Revista Química Nova na Escola, n.10, p.43-49, 1999. Disponível em: http://migre.me/uYuDW Acesso em 25 jan 2016.

GUAITA, R. I.; GONÇALVES, F. P. A experimentação na educação à distância: reflexões para a formação de professores de ciências da natureza. Em: XI Congresso Brasileiro do Ensino Superior à Distância, 2014, Anais... Florianópolis/SC: ESUD/UNIRED, p. 14611475 2014. Disponível em: http://migre.me/uYuJD Acesso em 20 jan. 2016.

HECKLER, V. Uso de simuladores e imagens como ferramentas auxiliares no ensino/aprendizagem de eletromagnetismo. Dissertação de Mestrado em Ensino de Física, Instituto de Física, UFRGS, Porto Alegre, 2004.

HEIDEMANN, L. A. et al. Ciclos de modelagem: uma proposta para integrar atividades baseadas em simulações computacionais e atividades experimentais no ensino de Física. Caderno Brasileiro de Ensino de Física, v. 29, n. 2 (Especial), p. 965-1007, out. 2012. Disponível em: $\quad$ https://periodicos.ufsc.br/index.php/fisica/article/view/21757941.2012v29nesp2p965/23071 Acesso em: 9 ago. 2015.

HONORATO, E. et al. Explorando uma aplicação m-learning para ensino de vetores na Física do ensino medio. In: SIMPÓSIO BRASILEIRO DE INFORMÁTICA NA EDUCAÇÃO, 26., 2015; CONFERÊNCIA LATINO-AMERICANA DE OBJETOS E TECNOLOGIAS DE 
APRENDIZAGEM, 10., 2015, Maceió. Anais... Maceió, 2015. Disponível em: http://www.brie.org/pub/index.php/sbie/article/view/5112/3517 Acesso em: 22 jan. 2016.

JARDIM, M. I. et al. Medições de atributos de ondas em uma cuba por meio de um software. In: ENCONTRO DE PESQUISA EM ENSINO DE FÍSICA, 12., 2010, Águas de Lindóia. Anais... Águas de Lindóia: Sociedade Brasileira de Física, 2010.

LIMA, J. F.; et al. Laboratório virtual: apresentação, conceituação, Análise e uma proposta de definição. XXIII Congresso Brasileiro de Ensino de Engenharia, Campina Grande PB, 12 - 15 set. 2005.

LUCENA, G. L. et al. Laboratório virtual como alternativa didática para auxiliar o ensino de química no ensino médio. Revista Brasileira de Informática na Educação, v. 21, n. 2, 2013. Disponível em: http://www.br-ie.org/pub/index.php/rbie/article/view/1427 Acesso em: 3 dez. 2014.

LUCERO, I. et al. Las TIC en la formación inicial docente de un profesorado de Física. In.: SILVA, J.; SALINAS, J. Innovando com TIC en la formación inicial docente: aspectos teóricos y casos concretos. Santiago de Chile: Enlaces, 2014. p. 99-116. Disponível em: https://www.academia.edu/13213630/Materiales curriculares digitales en la formaci\%C 3\%B3n inicial docente Acesso em: 21 jan. 2016.

MACÊDO, J. A.; DICKMAN, A. G. Simulações computacionais como ferramentas auxiliares ao ensino de conceitos básicos de eletricidade. Em: XVIII Simpósio Nacional de Ensino de Física, 2009, Anais... Vitória/ES: Sociedade Brasileira de Física, 2009.

MACINTYRE, A. B. Tecnologia e prazer: o ensino da matemática aplicada a administração. Dissertação de Mestrado em Engenharia de Produção, Universidade Federal de Santa Catarina, Florianópolis, 2002.

MARTINHO, T.; POMBO, L. Potencialidades das TIC no ensino das ciências naturais - um estudo de caso. Revista Electrónica de Enseñanza de las Ciencias, v. 8, no 2, p. 527-538, 2009. Disponível em: http://migre.me/uYuT4 Acesso em 20 mar 2016.

NEGROPONTE, Nicholas. A vida digital. São Paulo: Companhia das Letras, 1995.

OLIVEIRA, J. R. Contribuições e abordagens das atividades experimentais no ensino de ciências: reunindo elementos para a prática docente. Acta Scientiae, v.12, no 1, jan./jun. 2010, p. 141-146. Disponível em: http://migre.me/uYuXR Acesso em 25 jan 2016.

OURIQUE, P. A. et al. Fotografando estrelas com uma câmera digital. Revista Brasileira de Ensino de Física, v. 32, n. 1, 2010. Disponível em: http://www.sbfisica.org.br/rbef/pdf/ 321302.pdf Acesso em: 9 ago. 2015.

PINHO-ALVES, J. Atividades experimentais: do método prático à prática construtiva. Universidade Federal de Santa Catarina. Centro de Ciências da Educação. Programa de Pós Graduação em Educação. Doutorado em Educação: Ensino De Ciências Naturais. Tese de Doutorado. Florianópolis/SC, 2000. Disponível em: http://migre.me/uYv0g Acesso em 25 jan 2016. 
SAVI, R.; ULBRICHT, V. R. Jogos digitais educacionais: benefícios e desafios. RENOTE Revista Novas Tecnologias na Educação, v. 6, n. 2, dez., 2008. Disponível em: http://www.seer.ufrgs.br/renote/article/viewFile/14405/8310. Acesso em: 17 ago. 2015.

SILVA, I. P. Práticas experimentais mediadas pelas interfaces da internet na formação de professores de Física. Tese de Doutorado. Programa de Pós-graduação em Educação. Universidade Federal de Alagoas, 2016. Disponível em: http://www.repositorio.ufal.br/handle/riufal/1532 Acesso em 24 mar. 2017.

SIMÃO, J. P. et al. Utilização de experimentação remota móvel no ensino médio. Revista Novas Tecnologias na Educação, v. 11 n. 1, p. 1-11, jul. 2013. Disponível em: http://seer.ufrgs.br/index.php/renote/article/view/41701/26452 Acesso em: 22 jan. 2016.

SOUZA, A. L.; et al. Recursos da Computação Gráfica para o Desenvolvimento de um Laboratório Virtual de Teoria Eletromagnética. Em: XXIX Congresso Brasileiro de Educação em Engenharia, 2001, Anais... Porto Alegre/RS: PUCRS, 19 a 22 de dezembro de 2001. Disponível em: http://migre.me/uYv7T Acesso em 13 de set 2016.

SOUZA, A. M. Introdução a projetos de experimentos: caderno didático. UFSM, Santa Maria: 2002. Disponível em: http://migre.me/uYv8G Acesso em 25 jan 2016.

WILEY, D. Learning object design and sequencing theory. Tese de Doutorado. Doctor of Philosophy. Brigham Young University. 2000. Disponível em: http://migre.me/uYvaB Acesso em 21set 2015.

Submissão: 13/09/2016

Aceite: 29/01/2016 